\title{
Autonomous Building Detection Using Region Properties and PCA
}

\author{
Nour Aburaed*, Alavikunhu Panthakkan ${ }^{\dagger}$, Husameldin Mukhtar ${ }^{\dagger}$, \\ Wathiq Mansoor ${ }^{\dagger}$, Saeed Almansoori ${ }^{\ddagger}$ and Hussain Alahmad ${ }^{\dagger}$ \\ ${ }^{* \dagger}$ College of Engineering and IT, University of Dubai \\ $\ddagger$ Mohammed Bin Rashid Space Center (MBRSC) \\ Dubai, UAE \\ Email: *noaburaed@ud.ac.ae
}

\begin{abstract}
This paper proposes an algorithm for autonomous building detection in remote sensing images. The basis of the algorithm relies on the fact that each RGB channel conveys different information. Furthermore, region properties and Principal Component Analysis (PCA) are used to distinguish between buildings and other regions in order to reduce false positive cases. The images that are used to test the proposed algorithm are obtained from DubaiSat-2, which offers multispectral images with 1-m accuracy. The results of the algorithm indicate high accuracy and robustness against shadow effects.
\end{abstract}

Index Terms-Building Detection, Satellite Images, Edge Detection, Segmentation, Remote Sensing, PCA, Region Properties

\section{INTRODUCTION}

Satellite images and remote sensing methods are widely studied in the area of image processing due to the geospatial data that can be obtained from them, and the variety of practical applications that essentially rely on analyzing such images, which include city protection, geographical databases, military operations, and navigation. Obtaining information about earth's surface can, for instance, help to detect objects or make certain predictions about these objects with acceptable accuracy. Buildings are one example of such objects. Discussions regarding autonomous building detection as an open research problem have dominated research in recent years. This topic is concerned with detecting and isolating buildings using image processing and computer vision techniques with minimum human intervention. Most of the available tools allow building detection with a certain percentage of error, or with human intervention. However, with the rapid developments in urban areas, there are constant changes in buildings and other land features, which deem it impractical and costly to track these changes manually. Prior research generally confirms that, about a decade ago, building detection methods were not very effective due to the low resolution of the captured satellite images [1]. Nowadays, satellites can capture high resolution multispectral images, which provide enough spatial and spectral information in order to detect buildings. Examples of such satellites include QuickBird and IKONOS [1] [2] [3] [4] [5], as well as LIDAR [6] [7] [8], which provides 3D data that assist with detecting buildings using height information. However, the availability of high resolution images increases the inhomogeneity of the scene due to varying hierarchy and cluttered buildings, which makes extracting buildings one of the most challenging tasks [1]. Therefore, a reliable algorithm that detects buildings effectively and efficiently is needed. Several techniques have been reported in the literature. Some of those techniques include internal gray variance [2] [3], Convolutional Neural Networks [9], and Spectral Graph Theory [10], just to mention a few examples. This paper presents a method for autonomous building detection in remote sensing images obtained from DubaiSat-2. This satellite offers multispectral images of four bands; RGB and Near Infrared (NIR), with a resolution of 1-m/pixel. For more information about DubaiSat-2, the reader is referred to the paper [11] Manmade objects are easier to analyze using natural-color [1], therefore, the suggested algorithm relies on segmenting the image based on the different information conveyed by its RGB bands, along with region properties (also called features) and Principal Component Analysis (PCA) of each segment. This paper is divided as follows: Section II dissects the details of the algorithm, Section III demonstrates and evaluates the results of the algorithm, and finally, Section IV summarizes the paper and the future direction of this research.

\section{Methodology}

This section explores the details of the proposed autonomous building detection framework seen in Figure 1, which is divided into three main stages that will be explained in the next subsections. The image shown in Figure 2 will be used as an example throughout the paper to demonstrate the steps of the algorithm.

\section{A. Image Enhancement and Edge Detection}

In this stage, the image seen in Figure 2 is separated into its three RGB channels. Each channel is enhanced through contrast adjustment; the range of the channel is mapped to the intensity range [0 255]. In order to discard the noise while keeping the important information of the image, all three channels are binarized to different thresholds. The threshold level falls between $0-1$. If a pixel falls under the specified level, it is given a value of 0 . Otherwise, it is given a value of 1. Since blue is the channel that is the most susceptible to noise [12], the threshold is set to a high value that discards most of its pixels. On the other hand, the human eye is more sensitive 
Enhancement \& Edge Detection Segmentation and Labeling

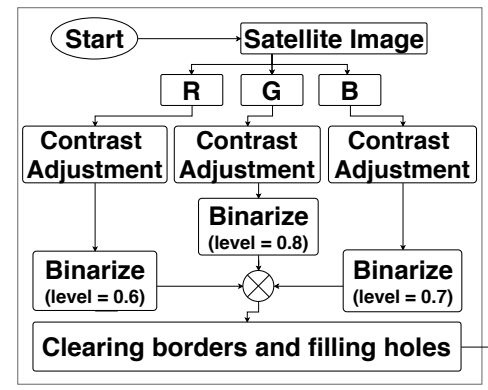

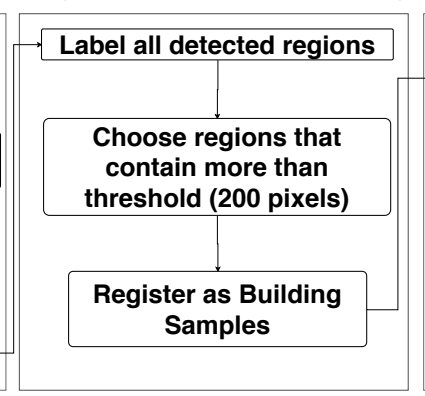

Classification

\begin{tabular}{|c|c|c|}
\hline $\begin{array}{c}\text { Compute features } \\
\text { and PCA for every } \\
\text { building region }\end{array}$ & $\begin{array}{l}\text { Compute features } \\
\text { and PCA for all the } \\
\text { detected regions }\end{array}$ & \multirow{2}{*}{$\begin{array}{l}\text { Compute Euclidean } \\
\text { Distance of PCA } \\
\text { between each region } \\
\text { and each building }\end{array}$} \\
\hline \multirow{2}{*}{\multicolumn{2}{|c|}{$\begin{array}{c}\text { Choose regions that fall within } \\
\text { Euclidean Distance of } \mathrm{PCA}<1.7 \text { and } \\
\text { features }<500000\end{array}$}} & \\
\hline & & \multirow{2}{*}{$\begin{array}{c}\text { Compute Euclidean } \\
\text { Distance of features } \\
\text { between each region } \\
\text { and each building }\end{array}$} \\
\hline & $\begin{array}{c}\text { Identify as } \\
\text { buildings }\end{array}$ & \\
\hline
\end{tabular}

Fig. 1. A flowchart of the proposed autonomous building detection algorithm.

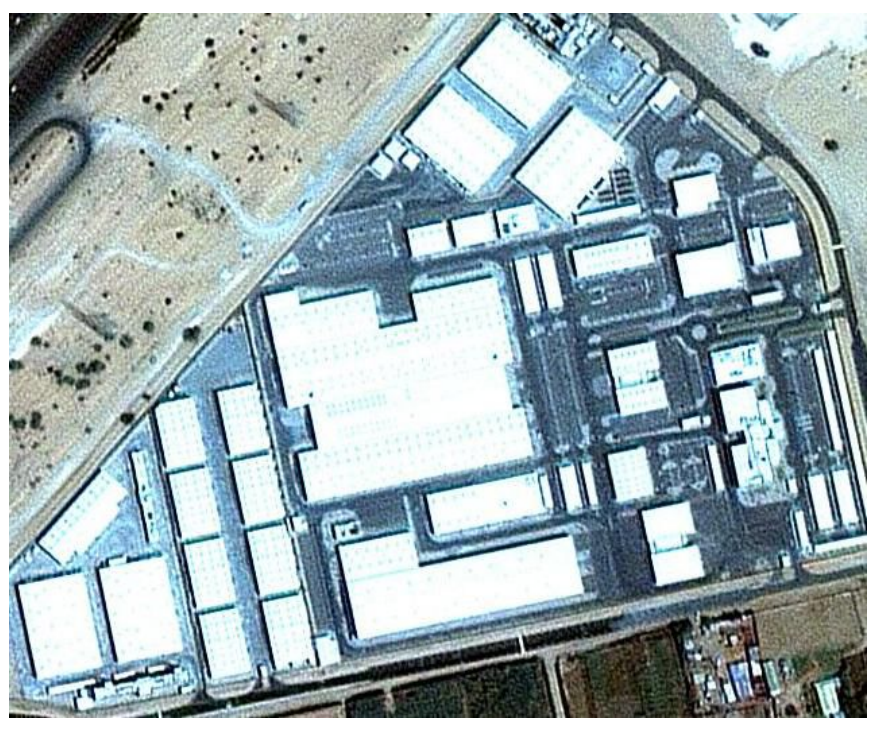

Fig. 2. An image of size $636 \times 523$.

to the green channel [13], however, the complexity of the scene will increase and it will become difficult to distinguish between buildings and other objects if the threshold is set to a low value. Therefore, it is set to a value that is lower than blue but higher than red. Finally, the threshold for red is set to a value around $0.5-0.6$ in order to balance out the noise coming from blue channel and overflow of information coming from green channel. As such, the chosen binarization thresholds for red, green, and blue channels are 0.6, 0.7, and 0.95, respectively. Logical AND is then performed across the three binarized channels in order to combine them. By using this logic, a pixel will retain a value of 1 only if all three channels agree that it is 1 , which asserts that it is an important piece of information that cannot be discarded. Figures 3(a) $3(\mathrm{e})$ show the results of applying all the aforementioned steps.

\section{B. Segmentation and Labeling}

In this stage, the image is segmented to regions based on the previously detected edges, as seen in Figure 3(f). Buildings vary in terms of shape and covered area. Thus, in order to identify all of them, the first step is to pick segments that are guaranteed to be buildings, and use their features as a

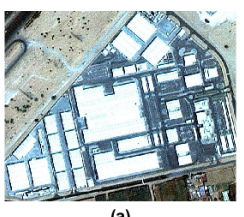

(a)

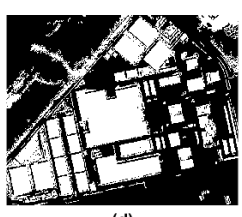

(d)

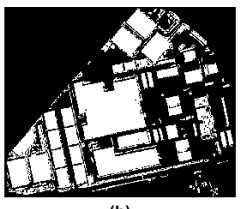

(b)

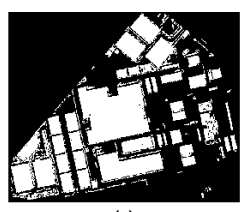

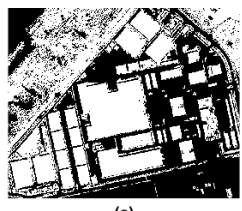

(c)

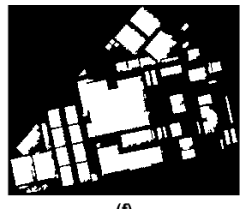

(f)
Fig. 3. This figure shows: (a) original $636 \times 523$ image; (b) binarized red channel; (c) binarized green channel; (d) binarized blue channel; (e) result of applying logical AND between (b), (c), and (d); lastly, (f) the final result after cleaning the borders and filling the holes in each connected region.

basis to detect the remaining buildings in the image. Since the precision of DubaiSat-2 represents $1 \mathrm{~m}$ by 1 pixel on the image, it can be assumed that regions that cover big areas in the image are buildings. To be more precise, the size of the area is decided based on a threshold, and any region bigger than this threshold is chosen as a building sample. Picking a threshold number that is too small or too big can lead to increase in False Positive (FP) or True Negative (TN) cases. Therefore, a threshold between 200 and 1000 can be considered realistic. For this demonstration, a threshold of 200 is chosen. The building samples that are chosen in this step will help to identify other buildings that might be too small or have complicated structures, as will be seen in the classification stage. Figure 4 shows the buildings that were chosen as samples marked on the image.

\section{Classification}

The next step in the algorithm is to compute the features matrix for the chosen building samples. The features matrix, which consists of twelve region properties, is recorded for each building region. Its region properties consist of Area, Convex Area, Filled Area, Perimeter, Eccentricity, Extent, Centroid, Major Axis Length, Minor Axis Length, Solidity, Orientation, and Equivalent Diameter. These twelve region properties give each building a unique PCA matrix. Summing up the coeffi- 


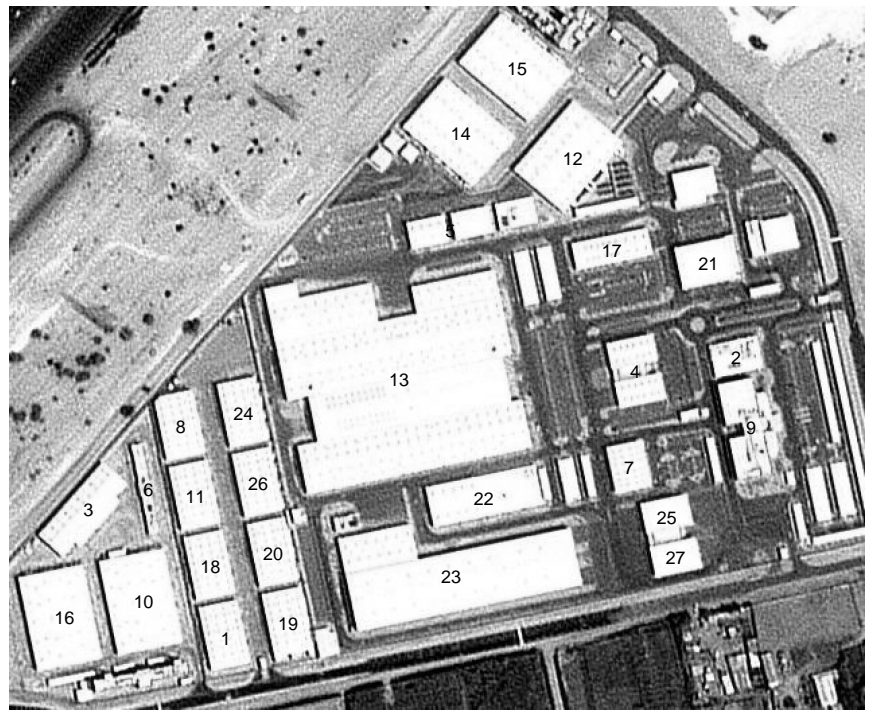

Fig. 4. The chosen building samples are marked on the image.

cients of this matrix gives a single, unique PCA value for each building. After this step, the algorithm computes a features matrix and PCA for every region in the image in a similar manner. The features matrices and PCA values that have been computed for building samples and regions will be compared using Euclidean Distance in order to detect the remaining buildings and reduce FP detections in the image according to a decision-making process. For each region in the image, the Euclidean distance between its features matrix and each features matrix of every building sample is computed. Only the minimum result is obtained. Similarly, the Euclidean distance between the region's PCA and the PCA of every building sample is computed, and the minimum one is obtained. Based on the observations from the images captured from DubaiSat2, the minimum Euclidean distance does not exceed 1.7 for PCA, and it does not exceed 5000000 for features matrix. Thus, these values are used as thresholds. In other words, for any region, if the minimum computed Euclidean distance between its PCA and that of building samples, and the minimum computed Euclidean distance between its features and that of building samples, do not exceed their respective thresholds, then the region is considered as a building. Figure 5 shows the result of the final stage of this algorithm. Changing PCA threshold by a difference of \pm 0.3 , or changing features threshold by a difference of \pm 10000 , does not cause any noticeable differences in the performance.

\section{RESUlts AND EVALUATION}

Quantifying an algorithm's results is important for benchmarking purposes and serves as a quality indicator of the algorithm's performance. While there is no official, standard method to evaluate building detection algorithms, the most frequently reported quantitative metrics in the literature are Detection Percentage (DP) and Branch Factor (BF). These

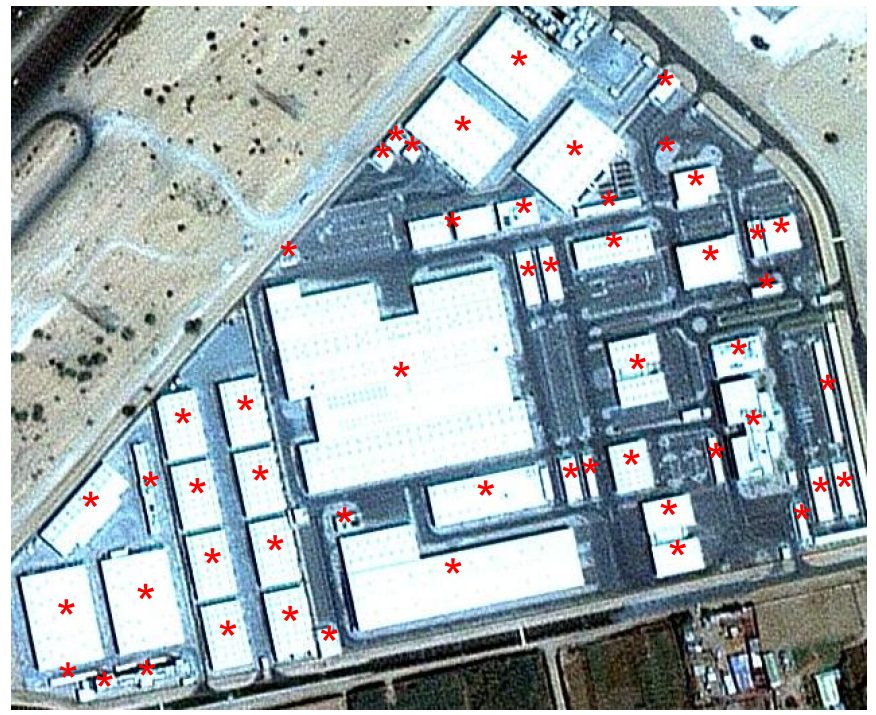

Fig. 5. All the detected buildings are marked on the image.

metrics were originally proposed by Lin and Nevatia [14], and they are defined as follows:

$$
\begin{aligned}
& D P=\frac{100 \times T P}{T P+T N}, \\
& B F=\frac{100 \times F P}{T P+F P},
\end{aligned}
$$

where True Positive (TP) indicates a building that is detected both manually and using the algorithm being evaluated, False Positive (FP) indicates a building that is detected by the algorithm but not manually, and True Negative (TN) indicates a building that is detected manually but not by the algorithm. Table I shows the results of applying the algorithm to different images taken from DubaiSat-2. Due to space limitations, only four results are shown. Additionally, $D P$ and $B F$ are computed and recorded in Table II. Since the framework of this algorithm relies on taking building samples in order to detect other buildings, the geometric complexity of a building's shape does not matter. Thus, any region that might be similar to a building, meaning that it falls within the specified PCA and features Euclidean Distance thresholds, is considered as a building.

\section{CONCLUSION}

In this paper, an autonomous building detection algorithm based on region properties and PCA has been developed and evaluated. Image segmentation is performed based on the amount of information conveyed by each RGB channel. Building samples are chosen from the segmented image as a basis to detect the remaining buildings in the image by using regions properties and PCA. The results of the algorithm strongly indicate high accuracy and effectiveness. In the future, this algorithm will be enhanced to make the thresholds more generalized, or controlled via GUI. Additionally, more restrictions will be imposed on picking building samples in 
TABLE I

RESUlts OF THE SUGgested Algorithm.

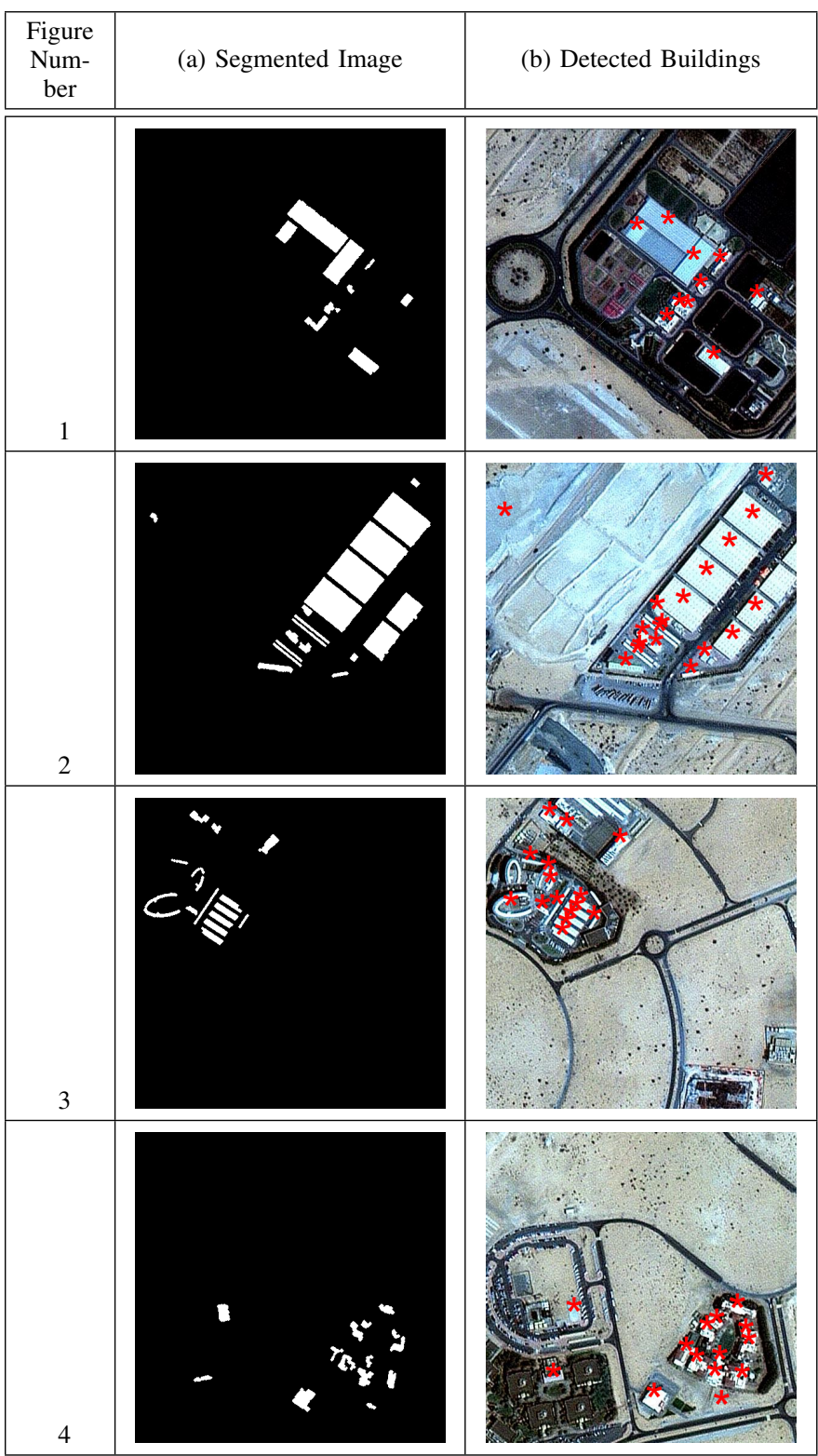

TABLE II

SUMMARY OF QUANTITATIVE EVALUATION RESULTS

\begin{tabular}{|c|c|c|c|c|c|}
\hline Figure Number & TP & FP & TN & DP (\%) & BF (\%) \\
\hline \hline 5 & 51 & 2 & 2 & 96.2 & 3.8 \\
\hline 1(b) in Table I & 10 & 0 & 0 & 100 & 0 \\
\hline 2(b) in Table I & 17 & 1 & 2 & 89.5 & 5.3 \\
\hline 3(b) in Table I & 13 & 1 & 1 & 92.9 & 7.1 \\
\hline 4(b) in Table I & 5 & 0 & 1 & 83.3 & 0 \\
\hline \hline Average DP & \multicolumn{5}{|c|}{$32.4 \%$} \\
\hline Average BF & \multicolumn{6}{|c|}{3.24} \\
\hline
\end{tabular}

order to prevent the possibility of choosing wrong samples. This can potentially be achieved by using localized gradient orientation. Texture analysis can also be used to enhance the process of classification.

\section{ACKNOWLEDGEMENT}

The authors would like to thank Mohammed Bin Rashid Space Center (MBRSC) for sponsoring and supporting this project.

\section{REFERENCES}

[1] L. Abraham and M. Sasikumar, "Structural feature extraction from satellite images," International Journal of Scientific \& Engineering Research, vol. 4, pp. 8-14, Dec. 2013

[2] D. Chaudhuri, N. K. Kushwaha, A. Samal, and R. C. Agarwal, "Automatic building detection from high-resolution satellite images based on morphology and internal gray variance," IEEE JOURNAL OF SELECTED TOPICS IN APPLIED EARTH OBSERVATIONS AND REMOTE SENSING, vol. 5, pp. 1767-1779, May 2016.

[3] A. Raikar and G. Hanji, "Automatic building detection from satellite images using internal gray variance and digital surface model," International Journal of Computer Applications, vol. 9, pp. 25-33, July 2016.

[4] K. Stankov and D. He, "Building detection in very high spatial resolution multispectral images using the hit-or-miss transform," IEEE GEOSCIENCE AND REMOTE SENSING LETTERS, vol. 10, no. 1, pp. 86-90, 2013.

[5] M. Wang, S. Yuan, and J. Pan, "Building detection in high resolution satellite urban image using segmentation, corner detection combined with adaptive windowed hough transform," in IEEE International Geoscience and Remote Sensing Symposium - IGARSS, (Melbourne, Australia), pp. 508-511, July 2013.

[6] F. Rottensteiner, J. Trinder, S. Clode, K. Kubik, and B. Lovell, "Building detection by dempster-shafer fusion of lidar data and multispectral aerial imagery," in Proceedings of the 17th International Conference on Pattern Recognition, vol. 2, pp. 339-342, Aug. 2004.

[7] R. Tse, C. Gold, and D. Kidner, "New approach to urban modeling based on lidar," in IEEE International Geoscience and Remote Sensing Symposium - IGARSS, (Plzen, Czech Republic), p. 279286, Feb. 2006.

[8] F. Tarsha-Kurdi, T. Landes, and P. Grussenmeyer, "New approach for automatic detection of buildings in airborne laser scanner data using first echo only," (Bonn, Germany), pp. 1-6, Sept. 2006.

[9] H. Miyazaki, K. Kuwata, W. Ohira, Z. Guo, X. Shao, Y. Xu, and R. Shibasaki, "Development of an automated system for building detection from high-resolution satellite images," in Fourth International Workshop on Earth Observation and Remote Sensing Applications, (Guangzhou, China), pp. 245-249, July 2016.

[10] A. Zakharov, A. Tuzhilkin, and A. Zhiznyakov, "Automatic building detection from satellite images using spectral graph theory," in International Conference on Mechanical Engineering, Automation and Control Systems (MEACS), (Tomsk, Russia), pp. 1-5, Dec. 2015.

[11] A. Bushahab, H. AlMatroushi, and S. AlMansoori, "Calibration and validation activities for dubaisat-2 performance assessment," in SPIE 9241, Sensors, Systems, and Next-Generation Satellites XVIII, (Amsterdam, Netherlands), Sept. 2014.

[12] P. Ganesan and V. Rajini, "Comparative study of denoising methods for satellite image restoration using matlab," International Journal of Advanced Research in Computer Science, vol. 4, pp. 74-44, May 2013.

[13] X. Tan, S. Lai, Y. Liu, and M. Zhang, "Green channel guiding denoising on bayer image," The Scientific World Journal, vol. 2014, pp. 1-9, Mar. 2014.

[14] C. Lin and R. Nevatia, "Building detection and description from a single intensity image," Comput. Vis. Image Underst., vol. 72, pp. 101-121, Nov. 1998. 